\title{
Kinetoplastid membrane protein-11 exacerbates infection with Leishmania amazonensis in murine macrophages
}

\author{
Daniel Ignacchiti Lacerda', Léa Cysne-Finkelstein', Marise Pinheiro Nunes, \\ Paula Mello De-Luca', Marcelo da Silva Genestra ${ }^{2+}$, Leonor Laura Pinto Leon², \\ Marcia Berrêdo-Pinho ${ }^{3}$, Leila Mendonça-Lima ${ }^{3}$, Denise Cristina de Souza Matos ${ }^{4}$, \\ Marco Alberto Medeiros ${ }^{5}$, Sergio Coutinho Furtado de Mendonça ${ }^{1 /+}$
}

\footnotetext{
'Laboratório de Imunoparasitologia ²Laboratório de Bioquímica de Tripanosomatídeos ${ }^{3}$ Laboratório de Genômica Funcional e Bioinformática, Instituto Oswaldo Cruz ${ }^{4}$ Laboratório de Tecnologia Imunológica ${ }^{5}$ Laboratório de Tecnologia Recombinante, Instituto de Tecnologia em Imunobiológicos-Fiocruz, Av. Brasil 4365, 21040-360 Rio de Janeiro, RJ, Brasil
}

In Leishmania amazonensis, kinetoplastid membrane protein-11 (KMP-11) expression increases during metacyclogenesis and is higher in amastigotes than in promastigotes, suggesting a role for this protein in the infection of the mammalian host. We show that the addition of KMP-11 exacerbates $\mathrm{L}$. amazonensis infection in peritoneal macrophages from BALB/c mice by increasing interleukin (IL)-10 secretion and arginase activity while reducing nitric oxide (NO) production. The doses of KMP-11, the IL-10 levels and the intracellular amastigote loads were strongly, positively and significantly correlated. The increase in parasite load induced by KMP-11 was inhibited by anti-KMP-11 or anti-IL-10 neutralising antibodies, but not by isotype controls. The neutralising antibodies, but not the isotype controls, were also able to significantly decrease the parasite load in macrophages cultured without the addition of KMP-11, demonstrating that KMP-11-induced exacerbation of the infection is not dependent on the addition of exogenous KMP-11 and that the protein naturally expressed by the parasite is able to promote it. In this study, the exacerbating effect of KMP-11 on macrophage infection with Leishmania is for the first time demonstrated, implicating it as a virulence factor in $\mathrm{L}$. amazonensis. The stimulation of IL-10 production and arginase activity and the inhibition of NO synthesis are likely involved in this effect.

Key words: kinetoplastid membrane protein-11 - Leishmania amazonensis macrophages - interleukin-10 - nitric oxide - arginase

The term leishmaniasis encompasses a complex of diseases characterised by clinical and epidemiological diversity. These diseases are broadly classified as tegumentary (affecting the skin, the facial mucosa or both) or visceral (a potentially fatal infection affecting lymphoid organs, such as the spleen, liver, bone marrow and lymph nodes) (Murray et al. 2005). These diseases are caused by protozoan parasites of the genus Leishmania, which have a life cycle with two stages: promastigote and amastigote. The flagellated, mobile promastigotes colonise the gut of the sandfly, where they undergo a process called metacyclogenesis (Sacks 1989). The infective metacyclic forms migrate to the foregut and from there into the proboscis of the insect. These metacyclic promastigotes are transmitted to the mammalian host by the bite of the insect and are taken up by mononuclear phagocytic cells. There, they transform into intracellular amastigotes, which multiply inside phagolysosomes, eventually lysing the cell to invade other mononuclear cells (Kane \& Mosser 2000).

Financial support: FIOCRUZ-PDTIS, IOC-FIOCRUZ, FAPERJ, CNPq

+ Corresponding author: mendonca@ioc.fiocruz.br

${ }^{\dagger}$ In memoriam

Received 3 August 2011

Accepted 7 December 2011
Leishmania amazonensis is a causative agent of New World cutaneous leishmaniasis. L. amazonensis infection leads to a spectrum of clinical presentations ranging from a single cutaneous lesion to diffuse cutaneous leishmaniasis, a clinical form that is usually refractory to treatment and is associated with the absence of Leishmania-antigen-specific cell-mediated immune responses (Convit et al. 1993). There is evidence that L. amazonensis is able to suppress the potentially effective immune responses of the host (Silveira et al. 2004) and that interleukin (IL)-10 contributes, at least in part, to the compromised parasite-specific immunity in L. amazonensisinfected hosts (Jones et al. 2002, Ji et al. 2003).

IL-10 is a cytokine produced by T cells, B cells, macrophages/monocytes and keratinocytes and it has broad anti-inflammatory properties. IL-10 can inhibit the synthesis of proinflammatory cytokines and chemokines as well as the production of nitric oxide (NO), superoxide anions and hydrogen peroxide (Spits \& de Waal Malefyt 1992, Opal et al. 1998, Moore et al. 2001), limiting the ability of macrophages to kill intracellular organisms (Frei et al. 1993, Reed et al. 1994, Murray et al. 1997, Kane \& Mosser 2001). NO is considered to be the principal effector molecule that mediates the intracellular killing of Leishmania amastigotes. Thus, the production of NO is critical for the control of Leishmania infection (Green et al. 1990, Soong et al. 1996). Some pathogens that infect macrophages, including Leishmania, induce IL-10 production 
as an immunosuppressive strategy for survival and replication (Belkaid et al. 2001, Redpath et al. 2001). IL-10 also induces the synthesis of arginase in murine macrophages (Iniesta et al. 2002). This enzyme catalyzes the synthesis of L-ornithine, which can be used by Leishmania to generate polyamines, essential growth factors for this parasite, leading to increased numbers of intracellular amastigotes (Iniesta et al. 2002, Kropf et al. 2005).

Kinetoplastid membrane protein-11 (KMP-11), a molecule that is present in all kinetoplastid protozoa (Stebeck et al. 1995), is a potent inducer of IL-10 production in peripheral blood mononuclear cells from patients with cutaneous leishmaniasis and it is also able to inhibit the interferon- $\gamma$ response of these cells to soluble Leishmania antigen stimulation (de Carvalho et al. 2003, Carvalho et al. 2005). Interestingly, it has been suggested that KMP-11 may play a role in the infectivity of Leishmania donovani promastigotes because the expression of this protein decreases in parallel with the loss of virulence associated with the repetition of subcultures (Mukhopadhyay et al. 1998).

These observations prompted us to investigate the effects of KMP-11 on the infection of murine macrophages with L. amazonensis. Our hypothesis was that KMP-11 would stimulate autocrine IL-10 secretion and increase parasite load.

\section{MATERIALS AND METHODS}

Mice - Female BALB/c mice, six-eight weeks old, were bred at the animal facilities of Oswaldo Cruz Foundation (Fiocruz), Brazilian Ministry of Health (MS). All mice were maintained under specific pathogen-free conditions. This research protocol was approved by the Ethical Committee for Animal Use of Fiocruz/MS (license L-0012/07).

Parasites - L. amazonensis (MHOM/BR/77/LTB0016) promastigotes provided by the Leishmania Collection of Fiocruz were grown at $25^{\circ} \mathrm{C}$ in Schneider's Drosophila medium (Sigma, St. Louis, USA) (pH 7.2) supplemented with $10 \%$ heat-inactivated foetal bovine serum (Invitrogen/ Gibco, Carlsbad, USA), 100 IU of penicillin, $100 \mu \mathrm{g} / \mathrm{mL}$ of streptomycin and $1.5 \mathrm{mM}$ L-glutamine (all from Sigma).

Recombinant L. amazonensis KMP-11 - Recombinant L. amazonensis KMP-11 was produced as described by Matos et al. (2010). The purified protein was analysed by $12.5 \%$ sodium dodecyl sulfate polyacrylamide gel electrophoresis (SDS-PAGE) and silver-nitrate staining, which indicated the absence of protein contaminants and the protein was identified with an anti-KMP-11 monoclonal antibody (Cedarlane, Burlington, Canada) with Western blot analysis. The N-terminal sequence of $L$. amazonensis KMP-11 was determined by direct amino acid sequencing (12 residues) using a Shimadzu Protein Sequencer (Model PSQ-1) equipped with an online phenylthiohydantoin amino acid analyser, as previously described (De-Simone et al. 2005), to confirm the identity of the protein. The levels of bacterial lipopolysaccharide (LPS) contamination in the recombinant protein preparation were determined using the Limulus amoebocyte lysate (LAL) test (BioWhittaker, Walkersville, USA) according to the manufacturer's recommendations. An LPS contamination of $0.13 \mu \mathrm{g} / \mathrm{mg}$ of protein was detected in the KMP-11 preparation. Before use in the experiments, LPS in the KMP-11 samples was removed using a polymyxin B-agarose column (Pierce Biotechnology, Rockford, USA) according to the manufacturer's instructions. After this procedure, no LPS contamination was detected by the LAL assay in the recombinant protein samples. The L. amazonensis KMP-11 preparation was adjusted to $1 \mathrm{mg} / \mathrm{mL}$ in PBS, aliquoted and stored at $-70^{\circ} \mathrm{C}$ until use.

Peritoneal macrophage cultures - Resident peritoneal macrophages were obtained from BALB/c mice by peritoneal washing with $5 \mathrm{~mL}$ of ice-cold, serum-free complete medium (RMPI-1640 medium supplemented with $1.5 \mathrm{mM}$ L-glutamine, $10 \mathrm{mM}$ HEPES, $100 \mathrm{IU} /$ $\mathrm{mL}$ penicillin, $100 \mu \mathrm{g} / \mathrm{mL}$ streptomycin, $5 \times 10^{-5} \mathrm{M}$ $\beta$-mercaptoethanol and $1 \mathrm{mM}$ sodium pyruvate) (all reagents purchased from Sigma). The cells were seeded on eight-well Lab-Tek chamber slides (Nalge Nunc International, Rochester, USA) at $4 \times 10^{5}$ cells in $400 \mu \mathrm{L}$ per well and allowed to adhere for $4 \mathrm{~h}$ at $37^{\circ} \mathrm{C}$ in $5 \% \mathrm{CO}_{2}$. Non-adherent cells were then removed by washing with complete medium at $37^{\circ} \mathrm{C}$. Cultures were kept overnight at $37^{\circ} \mathrm{C}, 5 \% \mathrm{CO}_{2}$, until the infection.

Infection of macrophages and incubation with KMP11 - Macrophage monolayers were infected with stationary-phase L. amazonensis promastigotes at a parasite to macrophage ratio of $1: 1$ at $32^{\circ} \mathrm{C}$ without or with KMP-11 $(10 \mu \mathrm{g} / \mathrm{mL})$ added $4 \mathrm{~h}$ after the addition of the parasites to the cultures. Immediately before the addition of the protein, the supernatants were harvested to remove extracellular parasites and the culture medium was replaced. These conditions were defined in preliminary kinetics and dose-response experiments, in which different doses of KMP-11, multiple parasite-to-macrophage ratios and various time points for the addition of KMP-11 were compared (data not shown).

Determination of intracellular parasite load and macrophage infection rate - Forty-eight hours after the addition of promastigotes, macrophage monolayers were washed twice in PBS, fixed with $100 \%$ methanol and stained with Giemsa. Intracellular parasite loads were assessed microscopically (100X, Axioskop, Carl Zeiss, Göttingen, Germany). At least 100 cells per Lab-Tek well were counted to determine the number of intracellular parasites and the percentage of infected macrophages (infection rate).

Measurement of IL-10 production - L. amazonensisinfected macrophages were treated or not with different doses of KMP-11 $(0.5,1,5$ or $10 \mu \mathrm{g} / \mathrm{mL}) 4 \mathrm{~h}$ after infection and the supernatants were harvested after $48 \mathrm{~h}$ of incubation. IL-10 levels were quantified by sandwich ELISA using a commercial kit (BD Biosciences Pharmingen, San Diego, USA) according to the manufacturer's instructions. For detection, streptavidin-alkaline phosphatase conjugate was used and the colour was developed with pnitrophenyl-phosphate (both from Zymed Laboratories, San Francisco, USA). The developed colour in the wells was measured at a wavelength of $450 \mathrm{~nm}$ using the VER$\mathrm{SA}_{\text {Max }}$ Microplate ELISA Reader (Molecular Devices, Sunnyvale, USA). Data were calculated in $\mathrm{pg} / \mathrm{mL}$ and the standard curve range was 30-4000 pg/mL. 
Treatment with neutralising antibodies - KMP-11 (10 $\mu \mathrm{g} / \mathrm{mL}$ ) was added to macrophage monolayers $4 \mathrm{~h}$ after infection with L. amazonensis with or without the simultaneous addition of $10 \mu \mathrm{g} / \mathrm{mL}$ of a neutralising anti-IL-10 monoclonal antibody (Stem RD, San Francisco, USA) or the same amount of an anti-KMP-11 monoclonal antibody (Cedarlane) or their isotype controls from the same manufacturers. All antibodies were mouse IgG1. Similar conditions of antibody treatment were also employed in macrophage monolayers without the addition of KMP-11.

Electrophoresis and immunoblotting - Supernatant proteins were obtained from macrophage cultures infected or not with L. amazonensis, as described above. The proteins were precipitated with $17 \%$ (v/v) trichloroacetic acid, washed with cold acetone and dissolved in a buffer containing $8 \mathrm{M}$ urea and 2\% CHAPS. Protein concentration was determined using the RC DC Kit (BioRad, Hercules, USA). Identical amounts of supernatant proteins $(20 \mu \mathrm{g})$ were loaded in each lane and resolved by $15 \%$ SDS-PAGE. Two micrograms of recombinant KMP-11 were loaded in parallel as a control and the gel was stained with Coomassie Brilliant Blue R-250. Proteins resolved in a replica gel were transferred to a nitrocellulose membrane (Hybond-C ${ }^{\mathrm{TM}}$ GE Healthcare) for $1 \mathrm{~h}$ at $100 \mathrm{~V}$ at $4^{\circ} \mathrm{C}$ with a Mini-TransBlot apparatus (Bio$\mathrm{Rad}$ ) and stained with a MemCode ${ }^{\mathrm{TM}}$ Reversible Protein Stain Kit (Pierce) for visualisation and subsequent quantitative analysis. The membrane was blocked overnight in 5\% non-fat milk in TBS, washed and incubated for $2 \mathrm{~h}$ with a mouse IgG1 anti-KMP-11 monoclonal antibody (1:3000 dilution) (Cedarlane). After washing, the membrane was incubated for $1 \mathrm{~h}$ with anti-mouse IgG HRPconjugated antibody (Pierce; 1:10000 dilution) followed by chemiluminescent detection using a Super Signal West Pico Kit (Pierce). The images were documented using a GS-800TM auto-calibrating imaging densitometer (Bio-Rad) and the relative molecular weights and intensities of the detected bands were analysed with Quantity One software version 4 (Bio-Rad).

Nitrite assay - Forty-eight hours after infection, supernatants were collected from the macrophage cultures. Nitrite, a stable oxidation product of $\mathrm{NO}$, was measured colourimetrically after adding Griess reagent $[0.1 \% \mathrm{~N}-(1-$ naphthyl)ethylenediamine in 5\% phosphoric acid and $1 \%$ sulphanilamide] in the same volume as the collected supernatants. After 10-15 min at room temperature (RT), the absorbance of the chromophore was measured at $540 \mathrm{~nm}$ using a VERSA ${ }_{\text {Max }}$ Microplate ELISA Reader. Nitrite concentrations were estimated by comparison with a standard curve $(0,1,5,10,25,50,75,90$ and $100 \mu \mathrm{M})$ prepared with sodium nitrite in complete medium (Green et al. 1982).

Determination of arginase activity - Arginase activity was measured as previously described (Corraliza et al. 1994) with some modifications. Briefly, $10^{6}$ cells were washed with a solution of $0.25 \mathrm{M}$ sucrose and $5 \mathrm{mM} \mathrm{KCl}$ and were added to $0.5 \mathrm{~mL}$ of $0.1 \%$ Triton $\mathrm{X}-100$ in an antiproteolytic buffer containing $0.1 \mathrm{mM}$ phenylmethylsulphonyl fluoride, $0.01 \%(\mathrm{w} / \mathrm{v})$ leupeptin, $0.2 \mathrm{mg} / \mathrm{mL}$ trypsin inhibitor and $1 \mathrm{mM}$ benzamidine in $0.25 \mathrm{M}$ sucrose and $5 \mathrm{mM} \mathrm{KCl}$. The mixture was then stirred for
$30 \mathrm{~min}$ at RT. After the cells were lysed, $0.5 \mathrm{~mL}$ of 25 $\mathrm{mM}$ Tris-HCL plus $5 \mathrm{mM} \mathrm{MnCl}$ ( $\mathrm{pH} 7.4$ ) was added and the enzyme was activated for $10 \mathrm{~min}$ at $56^{\circ} \mathrm{C}$. L-arginine hydrolysis was initiated by the addition of $25 \mu \mathrm{L}$ of $0.5 \mathrm{M}$ L-arginine ( $\mathrm{pH} 9.7$ ) to a $25-\mu \mathrm{L}$ aliquot of the previously activated lysate. After incubation at $37^{\circ} \mathrm{C}$ for $60 \mathrm{~min}$, the reaction was stopped by the addition of $400 \mu \mathrm{L}$ of an acid mixture containing $\mathrm{H}_{2} \mathrm{SO}_{4}, \mathrm{H}_{3} \mathrm{PO}_{4}$ and $\mathrm{H}_{2} \mathrm{O}$ (1:3:7). The amount of urea produced was measured by the addition of $25 \mu \mathrm{L}$ of $9 \% \alpha$-isonitrosopropiophenone (dissolved in $100 \%$ ethanol) and subsequent heating at $100^{\circ} \mathrm{C}$ for 45 min. After $10 \mathrm{~min}$ in the dark, the absorbance was determined at $540 \mathrm{~nm}$ in spectrophotometer (6405 Jenway, Dunmow, UK) using $200-\mu \mathrm{L}$ aliquots in a microculture plate. A calibration curve was prepared with increasing amounts of urea in a concentration range from 1.5-30 $\mu \mathrm{g}$ / $\mathrm{mL}$. All reagents were purchased from Sigma.

Statistical analysis - For each set of results, the data presented were collected from three independent experiments, each of them performed in triplicate, unless otherwise stated. The statistical analysis of the data was carried out using parametric (one-way ANOVA, followed by Tukey-Kramer multiple comparisons test, Student's $t$ test and linear regression) and nonparametric (Kruskal-Wallis test followed by Dunn's multiplecomparisons test, Mann-Whitney and Spearman's rank correlation) tests. Statistical significance was defined as $\mathrm{p}<0.05$ for all analyses. Similar results were obtained using parametric and non-parametric analyses. All tests were performed using GraphPad Prism 5 software (GraphPad Software, San Diego, USA).

\section{RESULTS}

Effect of KMP-11 on the infection of murine macrophages with L. amazonensis - The effect of the recombinant protein KMP-11 on parasite infectivity and/ or amastigote replication in vitro was investigated. Resident peritoneal macrophages from $\mathrm{BALB} / \mathrm{c}$ mice were infected with L. amazonensis and cultured with or without KMP-11. As shown in Fig. 1, $48 \mathrm{~h}$ after infection, the macrophage cultures containing KMP-11 presented significantly higher intracellular amastigote numbers and percentages of infected macrophages $(p<0.005$ in both cases) than those without KMP-11. These results were obtained with KMP-11 added $4 \mathrm{~h}$ after infection and after the removal of the remaining extracellular promastigotes by washing, demonstrating that the main effect of KMP-11 was to promote parasite survival/replication rather than to increase phagocytosis or promastigote infectivity. Therefore, we focused on the parasite load per macrophage rather than on the proportion of infected macrophages. Other recombinant proteins, namely bovine serum albumin and Megathura crenulata (keyhole limpet) haemocyanin (both purchased from Sigma), under the same experimental conditions, had no effect on the number of amastigotes per macrophage or on the macrophage infection rate (data not shown).

Correlations between doses of KMP-11, numbers of amastigotes and IL-10 levels - To provide evidence that the KMP-11-induced secretion of IL-10 is involved in the increase in the parasite load in L. amazonensis-infected mu- 
rine macrophages, we determined the correlations among the amount of KMP-11 added to the cultures, the number of amastigotes and the level of IL-10 secretion. Peritoneal macrophages were infected with $L$. amazonensis and then pulsed $4 \mathrm{~h}$ after infection with increasing KMP-11 doses $(0.5,1,5$ or $10 \mu \mathrm{g} / \mathrm{mL})$. The data shown in Fig. 2 represent the mean values of two independent experiments. There were strong and significant positive correlations between the added KMP-11 doses and the number of intracellular amastigotes (Fig. 2A) $(\mathrm{p}<0.01, \mathrm{r}=0.9834)$, between the doses of KMP-11 and the levels of IL-10 in the cell culture supernatants (Fig. 2B) $(\mathrm{p}<0.05, \mathrm{r}=0.9563)$ and between IL-10 levels and the numbers of amastigotes inside the macrophages (Fig. 2C) $(\mathrm{p}<0.05, \mathrm{r}=0.9257)$.

Neutralising antibodies for KMP-11 or IL-10 prevent the exacerbating effect of KMP-11 on the infection of murine macrophages with L. amazonensis - To further characterise the mechanism by which KMP-11 increases the parasite load in L. amazonensis-infected murine macrophages, as well as the role of IL-10 in this phenomenon, an anti-KMP-11 monoclonal antibody or a neutralising anti-IL-10 monoclonal antibody were added simultaneously with KMP-11. Fig. 3 shows the mean results \pm standard error of three independent experiments, each of them performed in duplicate. Both anti-KMP-11 and anti-IL-10 antibodies, but not their isotype controls, were able to significantly inhibit the increase in parasite load caused by the addition of KMP-11 (Fig. 3A) ( $p$ $<0.05$ ). Both anti-KMP-11 and anti-IL-10 antibodies, but not their isotype controls, were also able to significantly decrease parasite loads in macrophage cultures to which KMP-11 had not been added (Fig. 3B) $(\mathrm{p}<0.05)$, indicating that the KMP-11 already expressed by the parasite plays a role in exacerbating infection.

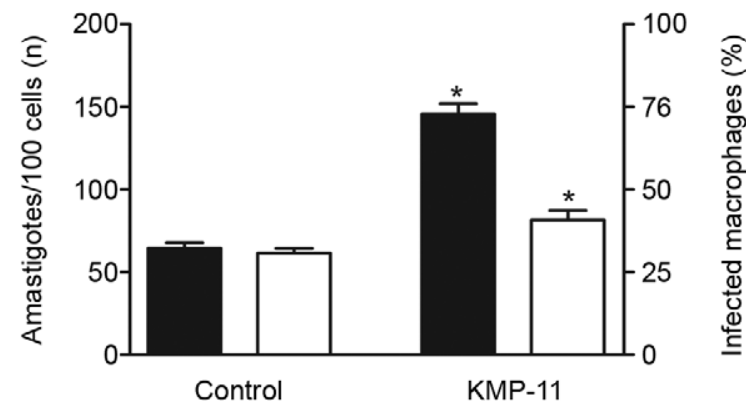

Fig. 1: effect of kinetoplastid membrane protein-11 (KMP-11) on the infection of peritoneal macrophages from BALB/c mice with Leishmania amazonensis. The cells were seeded on eight-well Lab-Tek chamber slides $\left(4 \times 10^{5}\right.$ cells in $400 \mu \mathrm{l}$ per well, stationary phase promastigote/ macrophage ratio of 1:1). Cultures were kept overnight at $37^{\circ} \mathrm{C}, 5 \% \mathrm{CO} 2$, until the infection. Forty-eight hours after infection, the macrophages monolayers were fixed and stained. Leishmania infection was assessed microscopically in terms of the numbers of amastigotes per 100 macrophages (black bars) and the percentages of infected macrophages (white bars) in absence (Control) or presence of KMP-11 $(10 \mu \mathrm{g} / \mathrm{ml})$ added $4 \mathrm{~h}$ after infection (KMP-11). Data are presented as the means of the results obtained in three independent experiments \pm standard error. Asterisk means $\mathrm{p}<0.005$ (significantly different from control).
$K M P-11$ is released in the supernatant of $L$. amazonensis-infected murine macrophage culture - After having shown that an anti-KMP-11 antibody was able to significantly decrease the parasite load in L. amazonensis-infected murine macrophages with or without the addition of KMP-11, adding further evidence that KMP11 promotes infection, it was necessary to understand how this antibody reaches its target, a membrane pro-
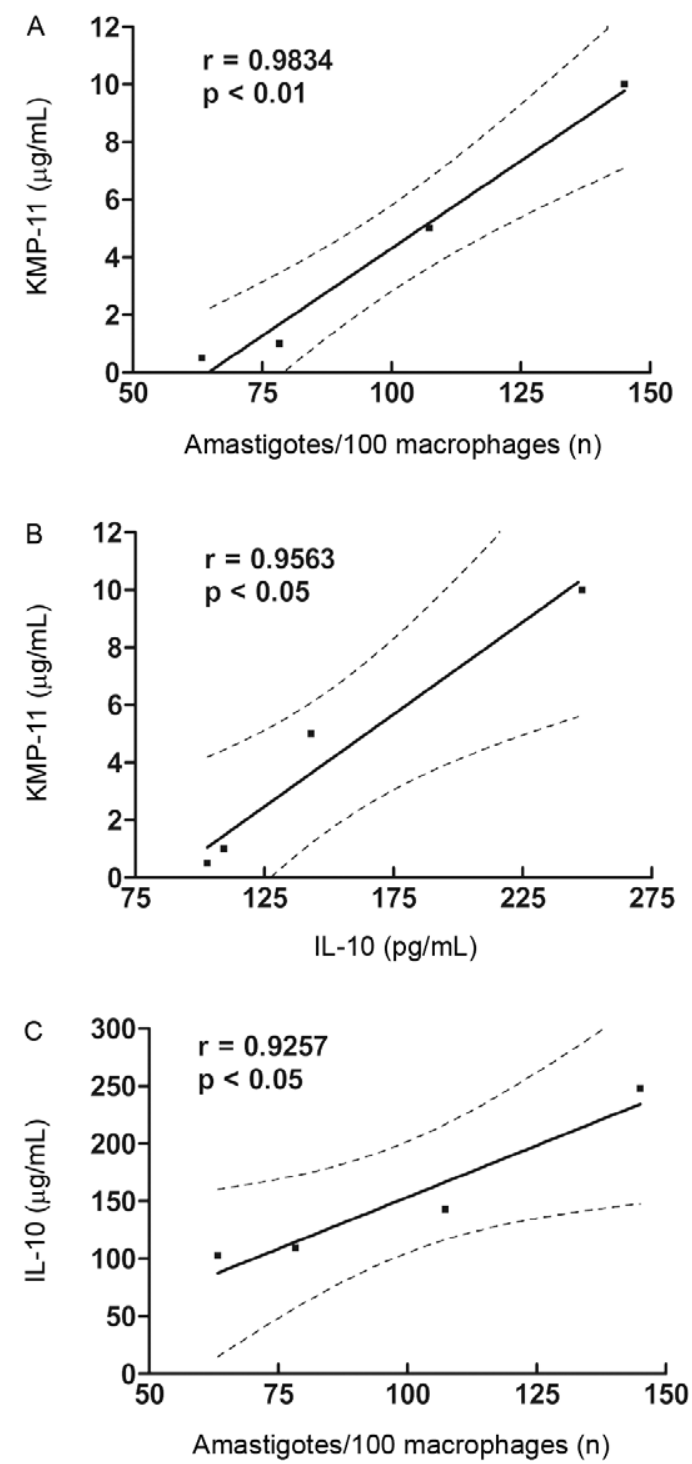

Fig. 2: correlations between kinetoplastid membrane protein-11 (KMP-11) doses, amastigote numbers and interleukin (IL)-10 levels in Leishmania amazonensis-infected peritoneal macrophages from $\mathrm{BALB} / \mathrm{c}$ mice. Macrophages were cultured and infected as described in the legend to Fig. 1 and several different KMP-11 doses were added $4 \mathrm{~h}$ after infection. Significant positive correlations were found between doses of KMP-11 added to the cultures and mean numbers of intracellular amastigotes per 100 macrophages (A), between doses of KMP-11 added to the cultures and IL-10 levels measured in the culture supernatants (B) and between IL-10 levels measured in the culture supernatants and mean numbers of intracellular amastigotes per 100 macrophages (C). The data shown represent the means of the results obtained from two independent experiments. 
tein of an intracellular parasite, in cultures without the addition of exogenous KMP-11. Therefore, we analysed the supernatant proteins obtained from non-infected or L. amazonensis-infected macrophage cultures with $15 \%$ SDS-PAGE. The proteins were transferred to a nitrocellulose membrane and visualised by MemCode ${ }^{\mathrm{TM}}$ staining. An 11-kDa band can be seen in the supernatant of $L$.
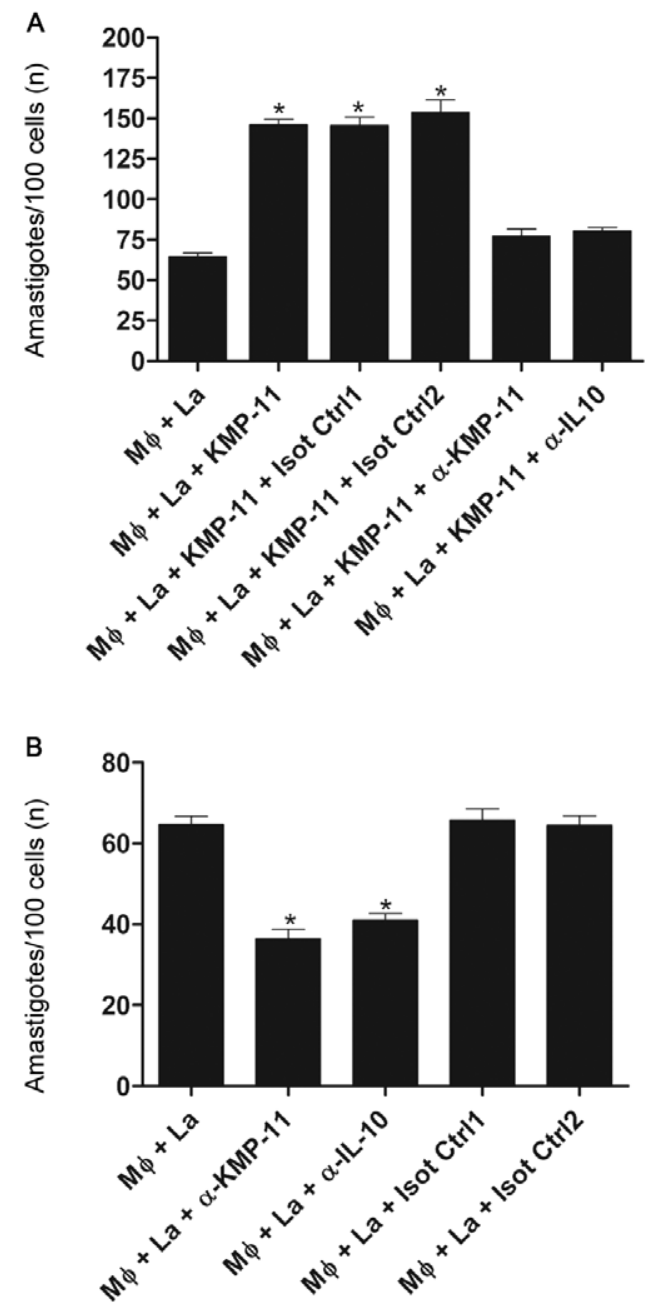

Fig. 3: inhibition of the exacerbating effect of kinetoplastid membrane protein-11 (KMP-11) on the infection of murine macrophages $(\mathrm{M} \varphi)$ with Leishmania amazonensis (La) by anti-KMP-11 and antiinterleukin (IL)-10 antibodies. M $\varphi$ were cultured and infected with La with (A) and without (B) addition of exogenous KMP-11, as described for Fig. 1, with or without $10 \mu \mathrm{g} / \mathrm{mL}$ of anti-KMP-11 ( $\alpha-\mathrm{KMP}-11)$ or neutralizing anti-IL-10 ( $\alpha$-IL-10) antibodies or yet isotype control antibodies (Isot Ctrl 1 or Isot Ctrl 2). Data are presented as the mean number of L. amazonensis amastigotes per $100 \mathrm{M} \varphi$ obtained in three independent experiments \pm standard error. The numbers of amastigotes in the cultures treated with KMP-11 alone or with KMP-11 plus the isotype controls were significantly higher than those of the cultures without KMP-11 and those of the cultures treated with KMP-11 plus anti-KMP-11 antibody or anti-IL-10 antibody (A). In absence of exogenous KMP-11, the treatment with anti-KMP-11 antibody or antiIL-10 antibody, but not with their isotype controls, significantly decreased the numbers of intracellular amastigotes (B). Asterisk means $\mathrm{p}<0.05$ as compared to the other conditions. Overall $\mathrm{p}$ value for multigroup comparison in $(\mathrm{A})<0.0001$ and in $(\mathrm{B})<0.0005$. amazonensis-infected macrophages, but not in the supernatant of non-infected macrophages (Fig. 4A). Immunoblotting analyses also revealed an $11-\mathrm{kDa}$ band that was recognised by a monoclonal anti-KMP-11 antibody in the supernatant of $L$. amazonensis-infected macrophages, but not in the supernatant of the uninfected macrophages (Fig. 4B). A second, minor immunoreactive band of $22 \mathrm{kDa}$, recognised by the anti-KMP-11 antibody in the recombinant KMP-11 preparation (Fig. 4B), is a dimeric form of the protein (Berberich et al. 1997, Ramírez et al. 1998). Taken together, the results presented in Fig. 4 demonstrate the presence of KMP-11 in the supernatants of $L$. amazonensis-infected macrophage cultures where the target antigen is readily accessible to the antibody.

The effect of KMP-11 on NO production by L. amazonensis-infected murine macrophages - Because NO plays a pivotal role in the killing of Leishmania amastigotes, we investigated whether the increase in parasite load caused by KMP-11 was accompanied by a decrease in NO production. Supernatants from cultures of $\mathrm{BALB} / \mathrm{c}$ mouse peritoneal macrophages infected or not with L. amazonensis and pulsed or not with KMP-11 (10 $\mu \mathrm{g} / \mathrm{mL}$ ) were assayed for nitrite concentration. L. amazonensis infection significantly increased nitrite production $(\mathrm{p}<0.001)$, but the addition of KMP-11 significantly decreased nitrite production $(p<0.001)$ to levels similar to those measured in the uninfected macrophage cultures. The addition of KMP-11 did not alter nitrite production in uninfected macrophage cultures (Fig. 5).
A

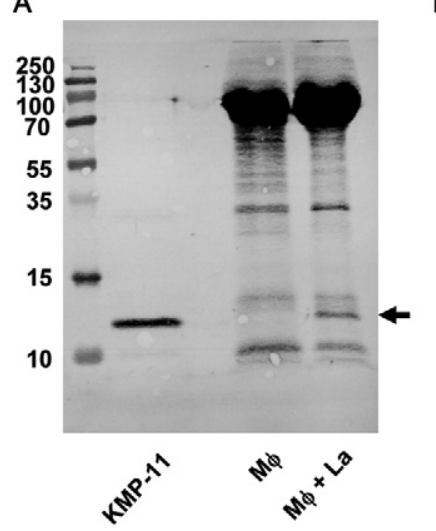

B

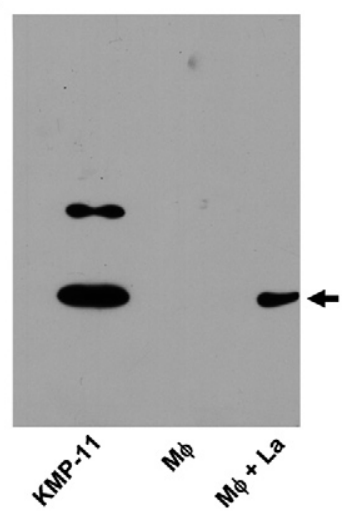

Fig. 4: kinetoplastid membrane protein-11 (KMP-11) is released in the supernatants of Leishmania amazonensis (La)-infected murine macrophage cultures. Twenty micrograms of supernatant proteins were obtained from macrophage cultures $(\mathrm{M} \varphi)$, infected or not with La, as described in the legend of Fig. 1, and resolved by $15 \%$ sodium dodecyl sulphate polyacrylamide gel electrophoresis (SDS-PAGE). Two micrograms of KMP-11 were loaded in parallel (KMP-11), as control. Proteins were transferred to a nitrocellulose membrane and stained with MemCode ${ }^{\mathrm{TM}}$ Reversible Protein Stain Kit (A). Immunoblotting analysis was performed with an anti-KMP-11 monoclonal antibody (B). Both SDS-PAGE (A) and immunoblotting (B) analyses revealed an $11 \mathrm{kDa}$ band (arrows) in the supernatant of La-infected macrophages, which is absent in the non-infected culture supernatant. The migration and size in $\mathrm{kDa}$ of molecular mass markers are indicated to the left of the figure. 
KMP-11 increases arginase activity in L. amazonensis-infected murine macrophages - Elevated arginase activity leads to increased amastigote loads in Leishmania-infected murine macrophages. Thus, we examined whether KMP-11 would trigger the arginase metabolic pathway. The addition of KMP-11 to cultures of L. amazonensis-infected peritoneal macrophages from BALB/c mice significantly increased arginase activity $(\mathrm{p}<0.001)$, as assessed by the urea levels in the cell lysates. However, the addition of KMP-11 was not able to increase arginase activity in cultures of uninfected macrophages (Fig. 6).

\section{DISCUSSION}

KMP-11 is found in all kinetoplastid protozoa (Stebeck et al. 1995) and is highly conserved (>95\% homology) in all Leishmania species, suggesting an essential role for this protein in the biology of the parasite (Ramírez et al. 1998). Recently, it has been demonstrated that the surface expression of this protein is higher in the amastigotes than in the promastigotes of L. amazonensis. Moreover, in L. amazonensis promastigotes, the surface expression of KMP-11 increases during metacyclogenesis (Matos et al. 2010). The increased expression of KMP-11 in metacyclic promastigotes and especially in amastigotes indicates a role for this molecule in the infection of the mammalian host. In this connection, KMP-11 expression has been associated with virulence in $L$. donovani promastigote cultures (Mukhopadhyay et al. 1998).

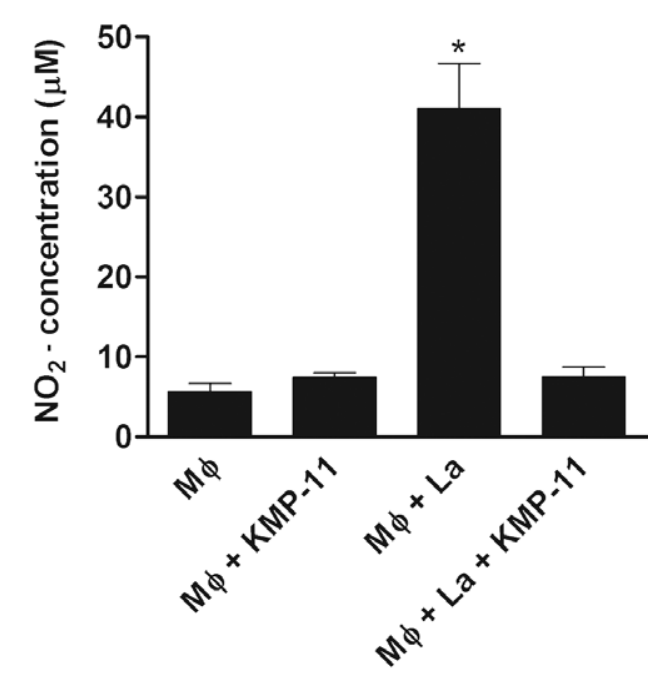

Fig. 5: kinetoplastid membrane protein-11 (KMP-11) inhibits nitric oxide (NO) production by BALB/c mice peritoneal macrophages $(\mathrm{M} \varphi)$. Supernatants from cultures of BALB/c mice peritoneal $\mathrm{M} \varphi$ infected or not with Leishmania amazonensis (La) in presence or absence of KMP-11, as described for Fig. 1, were assayed colorimetrically for nitrite production. KMP-11 $(10 \mu \mathrm{g} / \mathrm{mL})$ was added to the macrophage cultures $4 \mathrm{~h}$ after infection. The data shown represent the means \pm standard error of the results collected from three independent experiments. Asterisk means $\mathrm{p}<0.001$ as compared to uninfected $\mathrm{M} \varphi$ with or without KMP-11 and to infected M $\varphi$ with KMP-11. Overall $\mathrm{p}$ value for multigroup comparison $<0.0001$.
Here we show that KMP-11 is able to exacerbate the infection of $\mathrm{BALB} / \mathrm{c}$ mouse peritoneal macrophages with $L$. amazonensis by significantly increasing both the mean number of amastigotes inside the macrophages and the percentage of infected macrophages (Fig. 1). The fact that these results were obtained $4 \mathrm{~h}$ after infection and after the removal of the remaining extracellular promastigotes by washing demonstrates that the main infectionpromoting effect of KMP-11 was on parasite replication rather than on the internalisation of promastigotes. The higher proportion of infected macrophages would be a consequence of the heavier parasite burden, leading to an augmentation of host cell lysis, releasing more amastigotes and causing a larger propagation of the infection.

Evidence that the increase in amastigote replication induced by KMP-11 is associated with the stimulation of IL-10 production by the infected macrophages was provided by the strong, positive correlations between the KMP-11 dose and the number of intracellular amastigotes (Fig. 2A), between the KMP-11 dose and the level of IL-10 (Fig. 2B) and between the IL-10 level and the amastigote number (Fig. 2C). IL-10 has many strong macrophage-deactivating effects, deeply inhibiting a broad spectrum of macrophage/monocyte functions, including the synthesis of monokines such as IL-12, NO production and the expression of class II major histocompatibility complex (MHC) and co-stimulatory molecules such as CD80 and CD86 (Moore et al. 2001). In leishmaniasis, IL-10 plays a key role in the disease progression and promotes parasite persistence. IL-10 $/-\mathrm{BALB} / \mathrm{c}$ mice are able to control the disease progression, presenting smaller lesions and fewer parasites than their wild-type coun-

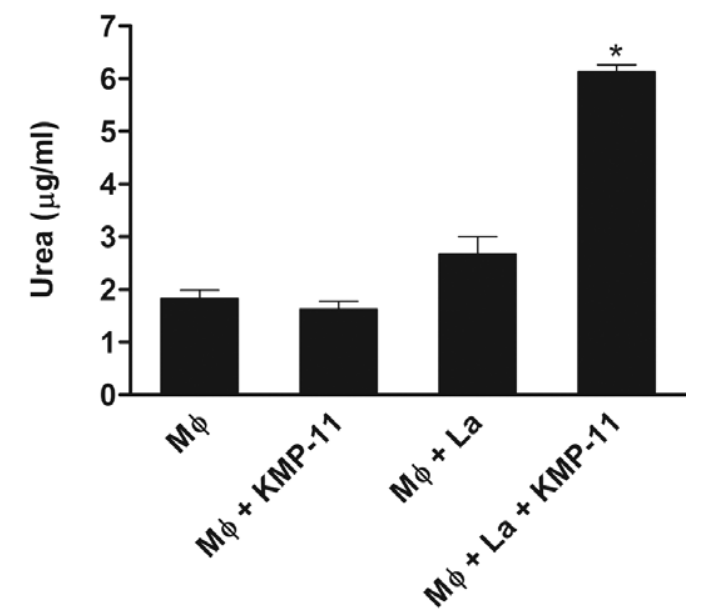

Fig. 6: Leishmania amazonensis (LA)-infected peritoneal macrophages $(\mathrm{M} \varphi)$ from $\mathrm{BALB} / \mathrm{c}$ mice present increased arginase activity after treatment with kinetoplastid membrane protein-11 (KMP-11). Cell lysates from cultures of BALB/c mice peritoneal $\mathrm{M} \varphi$ infected or not with La in presence or absence of KMP-11, as described for Fig. 1, were assayed colorimetrically for urea production. The data shown represent the means \pm standard error of the results collected from three independent experiments. Asterisk means $\mathrm{p}<0.001$ as compared to uninfected $\mathrm{M} \varphi$ with or without KMP-11 and to infected $\mathrm{M} \varphi$ without KMP-11. Overall p value for multigroup comparison $<0.0001$. 
terparts (Kane \& Mosser 2001) and IL-10-/- C57BL/10 (as well as C57BL/6 treated with anti-IL-10 receptor antibodies) are able to achieve sterile cure after infection with Leishmania major (Belkaid et al. 2001).

The exacerbation of L. amazonensis infection in murine macrophages caused by KMP-11 was further confirmed by blocking the effect of the addition of KMP-11 to the cultures through the simultaneous addition of an anti-KMP-11 monoclonal antibody (Fig. 3A). The involvement of IL-10 in this phenomenon was also confirmed by the abolishment of the KMP-11-induced increase in parasite load in L. amazonensis-infected macrophages provided by the addition of a neutralising anti-IL-10 monoclonal antibody (Fig. 3A). Isotype control antibodies were not able to inhibit the increase in the parasite load induced by the addition of KMP-11 to the macrophage cultures (Fig. 3A). Moreover, anti-KMP-11 was able to significantly decrease the mean parasite load in macrophage cultures that had not received exogenous KMP-11 (Fig. 3B), showing that even without the addition of exogenous KMP-11, the protein naturally expressed by the parasite, is also able to induce an increase in the number of intracellular amastigotes. A similar effect was obtained with the anti-IL-10 neutralising antibody (Fig. 3B), but not with the isotype controls. Considered together, our results confirm our hypothesis that KMP-11 promotes the exacerbation of L. amazonensis infection, increasing amastigote growth inside murine macrophages by inducing autocrine IL-10 secretion.

We have also demonstrated that the supernatants of L. amazonensis-infected murine macrophage cultures contain conspicuous amounts of KMP-11 (Fig. 4). The decrease in the parasite load caused by the addition of anti-KMP-11 antibodies into the macrophage cultures that had not received exogenous KMP-11 indicates that the protein that is released in the supernatant is, at least in part, responsible for the exacerbation of the infection. Thus, the addition of KMP-11 to the cultures was simply an amplification of something that occurs spontaneously during infection. The extracellular presence of KMP-11 in the supernatants of the Leishmaniainfected macrophage cultures is possibly a result of amastigote killing and lysis of parasitised macrophages (Chang et al. 2003). The generation of KMP-11-defficient mutants could help to determine the importance of this molecule for L. amazonensis infection. However, attempts to generate such mutants by the selective ablation of the KMP-11 genes have not been successful, likely because this protein is essential for the parasites' survival (El-Fadili et al. 2009).

NO production is an important effector mechanism for the killing of intracellular Leishmania amastigotes by infected macrophages (Green et al. 1990, Liew et al. 1990). The significant decrease in NO production in the L. amazonensis-infected macrophages treated with KMP-11 (Fig. 5) may also contribute to the increase in amastigote numbers in these macrophages (Fig. 2B). It has been shown that IL-10 can inhibit NO synthesis in human macrophages infected with Leishmania infantum and L. major (Vouldoukis et al. 1997). Moreover, IL-10 and other Th2 cytokines induce the expression and activity of arginase (Iniesta et al. 2002, Barksdale et al. 2004), driving L-arginine utilisation towards the production of polyamines, which are essential nutrients for Leishmania spp (Iniesta et al. 2002, Barksdale et al. 2004, Kropf et al. 2005, Wanasen \& Soong 2008). The addition of KMP-11 increased the arginase activity significantly in L. amazonensis-infected peritoneal macrophages from BALB/c mice (Fig. 6), which may also favour the enhancement of the parasite burden in these cells. In contrast to what was observed in the infected macrophages, KMP-11 had no effect on uninfected macrophages with respect to the induction of IL-10 secretion (data not shown), the inhibition of NO synthesis (Fig. 5) or the increase in arginase activity (Fig. 6).

Several studies have compared KMP-11 expression between different life cycle stages in some trypanosomatid species (Stebeck et al. 1995, Matos et al. 2010), but not between species. It would be interesting to know whether KMP-11 plays a similar role in the promotion of infection with other Leishmania species. We are currently investigating this possibility.

We have demonstrated that KMP-11 promotes amastigote growth in L. amazonensis-infected BALB/c macrophages. The exacerbation of the intracellular infection is likely a result of stimulation of IL-10 production, increase of arginase activity and inhibition of NO synthesis. Taken together, our results indicate that KMP-11 is a virulence factor in L. amazonensis, which makes this antigen a possible target for vaccines and therapeutic strategies.

\section{ACKNOWLEDGEMENTS}

To Dr Giovanni de Simone, for sequencing the amino terminus of L. amazonensis KMP-11, and to Rômulo J SoaresBezerra and Renan A Fernandes, for technical assistance.

\section{REFERENCES}

Barksdale AR, Bernard AC, Maley ME, Gellin GL, Kearney PA, Boulanger, BR, Tsuei BJ, Ochoa JB 2004. Regulation of arginase expression by T-helper II cytokines and isoproterenol. Surgery 135: 527-535.

Belkaid Y, Hoffmann KF, Mendez S, Kamhawi S, Udey MC, Wynn TA, Sacks DL 2001. The role of interleukin (IL)-10 in the persistence of Leishmania major in the skin after healing and the therapeutic potential of anti-IL-10 receptor antibody for sterile cure. J Exp Med 194: 1497-1506.

Berberich C, Requena JM, Alonso C 1997. Cloning of genes and expression and antigenicity analysis of the Leishmania infantum KMP-11 protein. Exp Parasitol 85: 105-108.

Carvalho LP, Passos S, Dutra WO, Soto M, Alonso C, Gollob KJ, Carvalho EM, Ribeiro de Jesus A 2005. Effect of LACK and KMP11 on IFN-gamma production by peripheral blood mononuclear cells from cutaneous and mucosal leishmaniasis patients. Scand J Immunol 61: 337-342.

Chang KP, Reed SG, McGwire BS, Soong L 2003. Leishmania model for microbial virulence: the relevance of parasite multiplication and pathoantigenicity. Acta Trop 85: 375-390.

Convit J, Ulrich M, Fernández CT, Tapia FJ, Cáceres-Dittmar G, Castés M, Rondón AJ 1993. The clinical and immunological spectrum of American cutaneous leishmaniasis. Trans $R$ Soc Trop Med Hyg 87: 444-448.

Corraliza IM, Campo ML, Soler G, Modolell M 1994. Determination of arginase activity in macrophages: a micromethod. $J$ Immunol Methods 174: 231-235. 
de Carvalho LP, Soto M, Jerônimo S, Dondji B, Bacellar O, Luz V, Orge Orge G, Alonso C, Jesus AR, Carvalho EM 2003. Characterization of the immune response to Leishmania infantum recombinant antigens. Microbes Infect 5: 7-12.

De-Simone SG, Correa-Netto C, Antunes OAC, De-Alencastro RB, Silva FP 2005. Biochemical and molecular modeling analysis of the ability of two p-aminobenzamidine-based sorbents to selectively purify serine proteases (fibrinogenases) from snake venoms. J Chromatogr B Analyt Technol Biomed Life Sci 822: 1-9.

El-Fadili K, Drummelsmith J, Roy G, Jardim A, Ouellette M 2009. Down regulation of KMP-11 in Leishmania infantum axenic antimony resistant amastigotes as revealed by a proteomic screen. Exp Parasitol 123: 51-57.

Frei K, Nadal D, Pfister HW, Fontana A 1993. Listeria meningitis: identification of a cerebrospinal fluid inhibitor of macrophage listericidal function as interleukin 10. J Exp Med 178: 1255-1261.

Green LC, Wagner DA, Glogowski J, Skipper PL, Wishnok JS, Tannenbaum SR 1982. Analysis of nitrate, nitrite and [15N] nitrate in biological fluids. Anal Biochem 126: 131-138.

Green SJ, Mellouk S, Hoffman SL, Meltzer MS, Nacy CA 1990. Cellular mechanisms of nonspecific immunity to intracellular infection: cytokine-induced synthesis of toxic nitrogen oxides from L-arginine by macrophages and hepatocytes. Immunol Lett 25: 15-19.

Iniesta V, Gómez-Nieto LC, Molano I, Mohedano A, Carcelén J, Mirón C, Alonso C, Corraliza I 2002. Arginase I induction in macrophages, triggered by Th2-type cytokines, supports the growth of intracellular Leishmania parasites. Parasite Immunol 24: 113-118.

Ji J, Sun J, Soong L 2003. Impaired expression of inflammatory cytokines and chemokines at early stages of infection with Leishmania amazonensis. Infect Immun 71: 4278-4288.

Jones DE, Ackermann MR, Wille U, Hunter CA, Scott P 2002. Early enhanced Th1 response after Leishmania amazonensis infection of C57BL/6 interleukin-10-deficient mice does not lead to resolution of infection. Infect Immun 70: 2151-2158.

Kane MM, Mosser DM 2000. Leishmania parasites and their ploys to disrupt macrophage activation. Curr Opin Hematol 7: 26-31.

Kane MM, Mosser DM 2001. The role of IL-10 in promoting disease progression in leishmaniasis. J Immunol 166: 1141-1147.

Kropf P, Fuentes JM, Fähnrich E, Arpa L, Herath S, Weber V, Soler G, Celada A, Modolell M, Müller I 2005. Arginase and polyamine synthesis are key factors in the regulation of experimental leishmaniasis in vivo. FASEB J 19: 1000-1002.

Liew FY, Li Y, Millott S 1990. Tumour necrosis factor (TNF-alpha) in leishmaniasis. II. TNF-alpha-induced macrophage leishmanicidal activity is mediated by nitric oxide from L-arginine. Immunology 71: 556-559.

Matos DCS, Faccioli LAP, Cysne-Finkelstein L, Luca PMD, CorteReal S, Armôa GRG, Lemes EMB, Decote-Ricardo D, Mendonça SCF 2010. Kinetoplastid membrane protein-11 is present in promastigotes and amastigotes of Leishmania amazonensis and its surface expression increases during metacyclogenesis. Mem Inst Oswaldo Cruz 105: 341-347.
Moore KW, de Waal Malefyt R, Coffman RL, O'Garra A 2001. Interleukin-10 and the interleukin-10 receptor. Annu Rev Immunol 19: 683-765.

Mukhopadhyay S, Sen P, Majumder HK, Roy S 1998. Reduced expression of lipophosphoglycan (LPG) and kinetoplastid membrane protein (KMP)-11 in Leishmania donovani promastigotes in axenic culture. J Parasitol 84: 644-647.

Murray HW, Berman JD, Davies CR, Saravia NG 2005. Advances in leishmaniasis. Lancet 366: 1561-1577.

Murray PJ, Wang L, Onufryk C, Tepper RI, Young RA 1997. T cellderived IL-10 antagonizes macrophage function in mycobacterial infection. J Immunol 158: 315-321.

Opal SM, Wherry JC, Grint P 1998. Interleukin-10: potential benefits and possible risks in clinical infectious diseases. Clin Infect Dis 27: 1497-1507.

Ramírez JR, Berberich C, Jaramillo A, Alonso C, Vélez ID 1998. Molecular and antigenic characterization of the Leishmania (Viannia) panamensis kinetoplastid membrane protein-11. Mem Inst Oswaldo Cruz 93: 247-254.

Redpath S, Ghazal P, Gascoigne NR 2001. Hijacking and exploitation of IL-10 by intracellular pathogens. Trends Microbiol 9: 86-92.

Reed SG, Brownell CE, Russo DM, Silva JS, Grabstein KH, Morrissey PJ 1994. IL-10 mediates susceptibility to Trypanosoma cruzi infection. J Immunol 153: 3135-3140.

Sacks DL 1989. Metacyclogenesis in Leishmania promastigotes. Exp Parasitol 69: 100-103.

Silveira FT, Lainson R, Corbett CEP 2004. Clinical and immunopathological spectrum of American cutaneous leishmaniasis with special reference to the disease in Amazonian Brazil - A review. Mem Inst Oswaldo Cruz 99: 239-251.

Soong L, Xu JC, Grewal IS, Kima P, Sun J, Longley BJ, Ruddle NH, McMahon-Pratt D, Flavell RA 1996. Disruption of CD40-CD40 ligand interactions results in an enhanced susceptibility to Leishmania amazonensis infection. Immunity 4: 263-273.

Spits H, de Waal Malefyt R 1992. Functional characterization of human IL-10. Int Arch Allergy Immunol 99: 8-15.

Stebeck CE, Beecroft RP, Singh BN, Jardim A, Olafson RW, Tuckey C, Prenevost KD, Pearson TW 1995. Kinetoplastid membrane protein-11 (KMP-11) is differentially expressed during the life cycle of African trypanosomes and is found in a wide variety of kinetoplastid parasites. Mol Biochem Parasitol 71: 1-13.

Vouldoukis I, Bécherel PA, Riveros-Moreno V, Arock M, da Silva O, Debré P, Mazier D, Mossalayi MD 1997. Interleukin-10 and interleukin-4 inhibit intracellular killing of Leishmania infantum and Leishmania major by human macrophages by decreasing nitric oxide generation. Eur J Immunol 27: 860-865.

Wanasen N, Soong L 2008. L-arginine metabolism and its impact on host immunity against Leishmania infection. Immunol Res 41: $15-25$. 